\title{
A REASONING COMPONENT'S CONSTRUCTION FOR PLANNING REGIONAL AGRICULTURAL ADVANTAGEOUS INDUSTRY DEVELOPMENT
}

\author{
Yue Fan ${ }^{1}$, Yeping Zhu ${ }^{1, *}$ \\ ${ }^{1}$ Agricultural Information Institute, Chinese Academy of Agricultural Sciences, Beijing, P. R. \\ China, 100081 \\ *orresponding author, Address: Library Room 310 Agricultural Information Institute, \\ Chinese Academy of Agricultural Sciences, Beijing 100081, P. R. China, Tel: +86-10- \\ 82105172,Fax: +86-10-82105172,Email: zhyp@mail.caas.net.cn
}
Abstract: The regional characteristic of China's agriculture and the present research conditions on regional agriculture have been studied first. After that, a new method is proposed that developing a system for planning regional agricultural advantageous industry development to help leaders and decision-making departments at all levels to make decisions. In order to achieve the construction of a reasoning component of such a system, the key technologies planned to be adopted are clarified in details, and the structure graph and functions graph of it have also been designed.

Key words: regional agricultural advantageous industry, reasoning component, forward chaining, knowledge presentation

\section{INTRODUCTION}

Compared with other industries, agriculture in our country bears obvious regional characteristic. China has a vast land area, throughout which land conditions vary quite differently, and different crops, different varieties and different planting management methods have to be altered from area to area. Thus an industry in a region shows an industrial advantage compared to other industries and regions. In order to reach the goal of high-quality, high-

Please use the following format when citing this chapter:

Fan, Y. and Zhu, Y., 2009, in IFIP International Federation for Information Processing, Volume 294, Computer and Computing Technologies in Agriculture II, Volume 2, eds. D. Li, Z. Chunjiang, (Boston: Springer), pp. 1291-1297. 
yield and high-efficiency in our country's agriculture development, detailed analysis of every industry in every region is indispensable. After knowing the advantageous industry in every region, its unique advantageous industry should merely be developed for one certain region, to ultimately fulfill the purpose of carrying out agricultural production by utilizing resources unique to their region.

At present, a great many scholars have been studying on the fundamental theories of regional agriculture, and a great deal economic analysis have been done, such as researches on comparative advantages of regional agricultural products, on regional agricultural competitiveness analysis, on regional agricultural industrial structure optimization, and on regional agricultural resources analysis. During these works, only a few information systems have been developed to assist analysis by using modern information technologies. These developed systems focus mostly on analysis of one industry or one kind of resource in some regions or on comparison of every industry in one region rather than on economic analysis and development planning of regional agriculture from the whole view and hardly on analysis of every kind of resources in one region. Thus it is necessary to develop a system for planning regional agricultural advantageous industry development to help leaders and decision-making departments at all levels to quickly, precisely, and adequately analyze developing conditions of regional agriculture, to understand regional agricultural production and development trends, and to make decisions. Such a system can comprehensively compare and analyze every industry and every kind of resources in every region from the nation's view, and can implement reasoning processes based on previous analysis results to get some reasoning results, through which to assist leaders make decisions.

This paper tries to study on the key technologies of constructing a reasoning component for planning regional agricultural advantageous industry development, clarifies some key technologies to be adopted, has done demand analysis, and completes the design of system structure graph and functions graph.

\section{KEY TECHNOLOGIES}

The research aims to develop a reasoning component to assist leaders make decisions on regional agricultural advantageous industry development planning. Thus component technology is firstly studied. In order to reason, knowledge on expert system is also studied, such as knowledge representation and storage, design of inference engine and inference processes. After that, such key technologies as COM and .NET component technology, building knowledge base based on database technology, and 
forward chaining are chosen to be adopted in the component's construction, and are detailed discussed as follows.

\subsection{COM and .NET component technology}

According to S. Kalaimagal and R. Srinivasan (2008), a component can be defined as "Any piece of independently executable binary code written to a specification, which can only be accessed via a set of well published interfaces and which can be integrated into any kind of software application irrespective of language/platform. A component always offers a set of services via its interfaces and may be encapsulated inside a container depending on the kind of middleware technology used to develop the component."

COM (Component Object Model) is a Microsoft centric interface standard for component-based software development introduced by Microsoft in 1993. The essence of COM is a language-neutral way of implementing objects such that they can be used in environments different from the one they were created in, even across machine boundaries. For well-authored components, COM allows reuse of objects with no knowledge of their internal implementation, as it forces component implementers to provide welldefined interfaces that are separate from the implementation. As the development of technology, COM has been one of the basic technologies integrated in Microsoft .NET, and the COM platform has also largely been superseded by the Microsoft .NET initiative. Users can easily develop COM components by using their familiar programming language in a .NET environment.

$\mathrm{COM}$ is very similar to other component software interface standards, such as CORBA and Java Beans, although each has its own strengths and weaknesses. It is likely that the characteristics of COM make it most suitable for the development and deployment of desktop applications, for which it was originally designed. Hence Microsoft .NET is adopted in this developing processes.

\subsection{Building knowledge base based on database}

The database technology bears such characteristics as structured data, low redundancy, high independence, easy expandability, and the non-procedural usage of data and shared data. As the development of database technology, especially the increasingly powerful functions and calculation speed of relational database, its application is more and more extensive.

Knowledge text files are usually adopted in traditional knowledge base management. A file in a system serves specially for a certain application, 
and data's logical structure is the best for it. This leads to the bad condition when database alters that application program and the definition of file structure must be adapted to data's logical structural alterations. Thus it lacks independence between database and application program in this way. This method also cannot meet the demands of sharing data between several users or programs and has difficulty in database maintaining.

Both differences and similarities exist between a database and a knowledge base, while the former manages and stores data, and the latter focuses on the management and storage of knowledge. A database manages information data, while a knowledge base processes intelligent information. A database tries to implement effective storage and search from large amount of data, while a knowledge base to fulfill effective knowledge representation and reasoning mechanism. The similarities between them lie in their realization that rules and facts in one knowledge base could be built in form of database tables to be easily added, altered, deleted or queried. Hence the combination of database and knowledge base, which combines factual data with abstract knowledge, and processing capability of data with that based on knowledge, could contribute a lot to the construction of a more effective knowledge base system.

In this realization, knowledge is represented in the general form of production rules: $\sigma_{1}+\sigma_{2}+\sigma_{3} \rightarrow \Omega$, that is: when precondition $\sigma_{1}, \sigma_{2}$, and $\sigma_{3}$ are met at the same time, action $\delta$ is triggered. Rules are stored in the form of rule tables in the database. Each table is composed of elements of such fields as Rule No., Precondition 1, Precondition 2, Precondition 3, Action, Interpretation Rule No., as follows:

Table 2. A production rules' table

\begin{tabular}{lccccc}
\hline Rule No. & Precondition 1 & Precondition 2 & Precondition 3 & Action & Interpretation Rule No. \\
\hline 1001 & $\begin{array}{c}\text { Yield of wheat }> \\
10 \mathrm{t}\end{array}$ & $\begin{array}{c}\text { Yield of maize } \\
<1 \mathrm{t}\end{array}$ & $\begin{array}{c}\text { Better to } \\
\text { plant wheat }\end{array}$ & 001 \\
$\ldots$ & & & & $\ldots$ \\
\hline
\end{tabular}

\subsection{Forward chaining}

There are two main methods of reasoning when using inference rules: backward chaining and forward chaining.

Forward chaining starts with the available data and uses inference rules to extract more data (from an end user for example) until an optimal goal is reached. An inference engine using forward chaining searches the inference rules until it finds one where the antecedent (If clause) is known to be true. When found it can conclude, or infer, the consequent (Then clause), resulting in the addition of new information to its data. Inference engines will often cycle through this process until an optimal goal is reached. 
Because the data determines which rules are selected and used, this method is called data-driven, in contrast to goal-driven backward chaining inference.

One of the advantages of forward-chaining over backward-chaining is that the reception of new data can trigger new inferences, which makes the engine better suited to dynamic situations in which conditions are likely to change.

\section{SYSTEM DESIGN}

\subsection{System structure graph}

Based on forward chaining discussed before, the system structure graph of this reasoning component has been designed. It is designed into three levels: Graphical user interface level、Inference engine realization level、 and Data maintenance level.

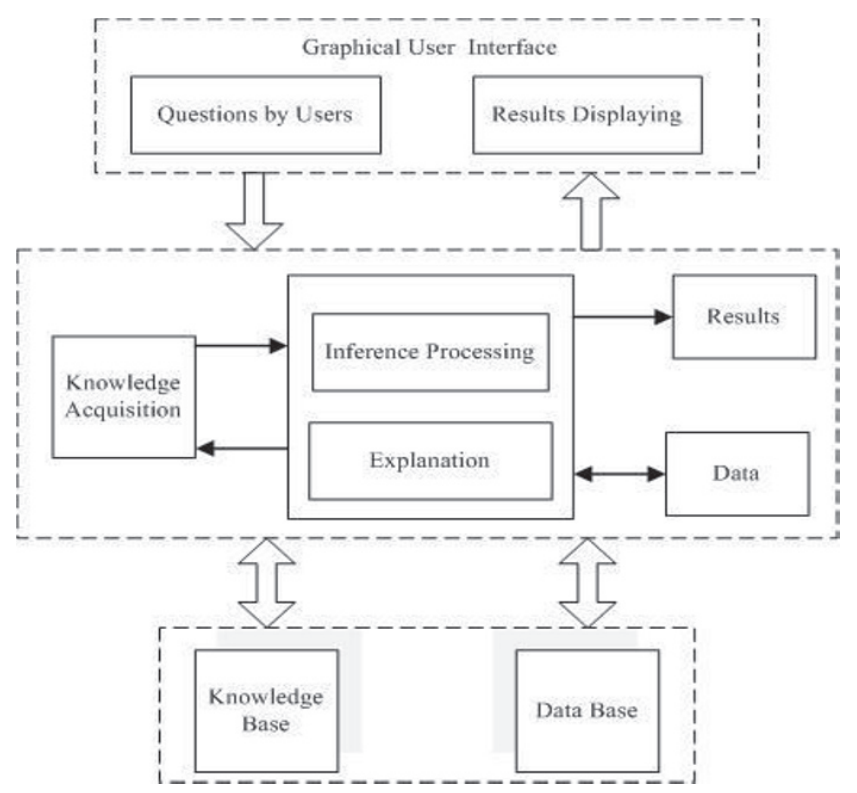

Figure 1. System structure graph

By adopting visual programming language, graphical user interface level fulfills the interaction between program and users, such as the questions raised by users, the program required data input of users to initiate inference, the inference results displaying, and etc. 
The middle level is critical to the component, which accomplishes the whole inference engine based on forward chaining. It achieves the realization of the whole inference processes, including the implements of explanation mechanism, production rules' selection and inference, the feedback data's input, and so on.

The data level manages and maintains data base and knowledge base. Knowledge's representation and storage, design of data tables and knowledge tables, data's alteration and edit are all implemented here.

\subsection{Functions graph}

The component's functions graph is also designed here. It is divided into four parts: Data alteration、Inference and planning System management and Help. Details are depicted in the following figure:

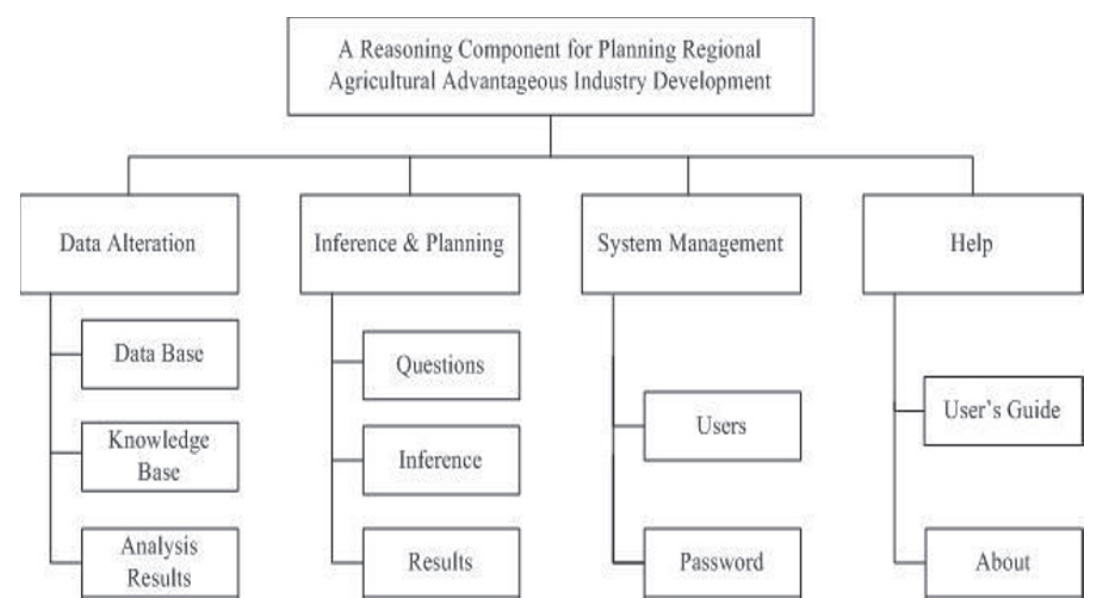

Figure 2. Functions graph

\section{CONCLUSION}

After continual studies, key technologies of the component have been chosen. And after demand analysis, system structure graph and functions graph of it have also been designed as mentioned above. The following works will focus on realization of the component and improvement of its functions, the obstacles confronted will be overcome after continuing studying and researches. 


\section{ACKNOWLEDGEMENTS}

This research was supported by National Scientific and Technical Supporting Programs Funded by State High-tech Research and Development Project of China (2007AA10Z237 , 2006BAD10A06), and Beijing Municipal Natural Science Foundation (4042026).

\section{REFERENCES}

http://en.wikipedia.org/wiki/Main_Page

S. Kalaimagal, R. Srinivasan. A TRUSTAD Component Nomenclature, Journal of Object Technology, Vol.7 No.4, Issue May-June 2008, pp 159-173

Wang Wei, He Jianjun. Establishing of Expert System for Pneumatic Drying Process Based on Database Technology, Automation \& Instrumentation, 2007(3): 9-11, 72(in Chinese)

Yang Changbao, Wu Xiuyuan, Ma Shengzhong. The Study of Intelligent GIS and Its Application in Info-Agriculture, Journal of Jilin Agricultural University, 2004, 26(1): 111 115(in Chinese) 